Advocates say xenotransplantation could save thousands of lives each year by providing organs for people who otherwise would die waiting. By the end of 1994, more than 37,000 patients were on the waiting list for donated organs. Approximately $50 \%$ of the patients on the waiting list die before a suitable organ becomes available.

Guidelines are being developed by the FDA and the $\mathrm{CDC}$. The IOM committee also recommends that an advisory committee with Health and Human Services be created to coordinate development, oversight, and evaluation of guidelines among federal agencies and other institutions conducting clinical trials. In addition, the IOM committee recommends that guidelines for human clinical trials address four major areas to prevent animal-to-human infection: (1) screening donor animals for infectious organisms and considering the development of specific pathogen-free animals; (2) lifetime surveillance of patients for infectious diseases and periodic surveillance of their families and healthcare workers; (3) establishment of tissue banks for blood and tissue samples from source animals and patients; and (4) establishment of local and national registries of xenotransplant patients, in coordination with international registries.

The committee also considered the possibility of raising animals in a germ-free environment to reduce the risk of infection. The committee concluded that, while the expense to implement the recommendations may be substantial, "the potential of xenotransplantation is great enough to justify funding by federal agencies, private industry, and other sources of research and other programs necessary to minimize the risk of disease transmission."

FROM: Institute of Medicine. Xenograft transplantation: science, ethics, and public policy. Report: Xenograft. July 17, 1996.

\section{AMA Guide on AIDS}

After years of emphasizing early detection and treatment of HIV infection, the American Medical Associa- tion (AMA) is asking primary care physicians to expand their scope of care with more comprehensive prevention efforts. The AMA, with support from the Henry J. Kaiser Foundation, is sending out a new guide on AIDS education for patients to every US family practitioner, internist, pediatrician, and gynecologist. The guide offers specific directions for helping patients to assess their lifestyle risks and to make choices aimed at infection prevention.

The bulk of the information in the guide focuses on two primary risk factors-sexual behavior and injection drug use. It includes, for example, a section on how to instruct injection drug users to sterilize needles-a discussion that may conflict with some physicians' beliefs about drug use. Dr. James Allen, an AMA public health expert, said the goal is to keep such users from getting AIDS while trying to get them into treatment. The guide also contains a list of risk facts associated with specific sexual practices that the physician can use to objectively discuss HIV prevention and reinforce safer sexual behaviors. There is an illustrated section that provides instruction on how to use a condom.

In addition, the guide emphasizes that healthcare workers must avoid verbal and nonverbal messages that convey philosophical, religious, or moral views about intravenous drug use or sexual behavior.

The AMA said they hope to get the guide out to 200,000 physicians by the end of the year.

FROM: AMA releases explicit guide on discussing AIDS. Chicago Tribune July 7, 1996;1:3.

Voelker R. Medical news and perspectives: HIV guide for primary care physicians stresses patient-centered prevention. JAMA 1996;276:85-86.

Additional news items in this issue: CDC Restricts Transfer of Biohazards, page 572; HIV Origins, page 594; Prevalence of $\mathrm{C}$ difficile, page 616; 9,000 E Coli Cases in Japan, page 622. 